OPEN ACCESS

Check for updates

\title{
National, regional, and worldwide epidemiology of psoriasis: systematic analysis and modelling study
}

\author{
Rosa Parisi, ${ }^{1,2}$ Ireny Y K Iskandar, ${ }^{1}$ Evangelos Kontopantelis, ${ }^{2}$ Matthias Augustin, ${ }^{3}$ \\ Christopher E M Griffiths, ${ }^{4,5}$ Darren M Ashcroft, ${ }^{1,5}$ on behalf of the Global Psoriasis Atlas
}

${ }^{1}$ Division of Pharmacy and Optometry, University of

Manchester, Manchester, UK

${ }^{2}$ Division of Informatics,

Imaging \& Data Sciences,

University of Manchester,

Manchester, UK

${ }^{3}$ Institute of Health Care

Research in Dermatology and Nursing, University Medical

Centre Hamburg-Eppendorf,

Hamburg, Germany

${ }^{4}$ Dermatology Centre, Salford Royal NHS Foundation Trust.

Manchester, UK

${ }^{5} \mathrm{NIHR}$ Manchester Biomedical Research Centre, University of Manchester, Manchester, UK

Correspondence to: R Parisi, Division of Informatics, Imaging \&

Data Sciences, School of Health

Sciences, University of Manchester, Manchester M13 9PT, UK

rosa.parisi@manchester.ac.uk (or @RosaParisi09 on Twitter ORCID 0000-0002-0968-9153)

Additional material is published online only. To view please visit the journal online.

Cite this as: $B M J$ 2020;369:m1590 http://dx.doi.org/10.1136/bmi.m1590

Accepted: 30 March 2020

\section{ABSTRACT}

OBJECTIVE

To systematically review and provide information on the incidence of psoriasis and quantify global, regional, and country specific estimates of its prevalence.

DESIGN

Systematic review and meta-analysis.

\section{DATA SOURCES}

Medline, Embase, Web of Science, SciELO, Korean Journal Databases, Russian Science Citation Index, WPRIM, SaudiMedLit, Informit, IndMed, and HERDIN were searched systematically from their inception dates to October 2019.

\section{METHODS}

Studies were included if they reported on the incidence or prevalence of psoriasis in the general population. Incidence data were summarised descriptively, whereas bayesian hierarchical models were fitted to estimate the global, regional, and country specific prevalence of psoriasis.

\section{RESULTS}

41164 records were identified and 168 studies met the inclusion criteria. In adults, the incidence of psoriasis varied from 30.3 per 100000 person years ( $95 \%$ confidence interval 26.6 to 34.1 ) in Taiwan to 321.0 per 100000 person years in Italy. The prevalence of psoriasis varied from $0.14 \%$ ( $95 \%$ uncertainty interval $0.05 \%$ to $0.40 \%$ ) in east Asia to $1.99 \%(0.64 \%$ to $6.60 \%)$ in Australasia. The prevalence of psoriasis was also high in western Europe (1.92\%, $1.07 \%$ to $3.46 \%$ ), central Europe (1.83\%, $0.62 \%$ to $5.32 \%)$, North America (1.50\%,

\section{WHAT IS ALREADY KNOWN ON THIS TOPIC}

Psoriasis is a chronic, disabling skin disease associated with psychological, metabolic, arthritic, and cardiovascular comorbidities

In 2014, the World Health Organization recognised psoriasis as a serious noncommunicable disease and its report called for greater understanding of the epidemiology of the disease

The Global Burden of Disease group has produced estimates on the prevalence of psoriasis for 21 regions of the world but not for individual countries

\section{WHAT THIS STUDY ADDS}

This systematic review and meta-analysis provides global, regional, and country specific estimates of the prevalence of psoriasis

Psoriasis is a common disease that mainly affects the adult population, and is more frequent in high income countries

An improved understanding of the epidemiology of psoriasis is important when allocating resources to reduce morbidity, disability, and mortality associated with the disease
$0.63 \%$ to $3.60 \%$ ), and high income southern Latin America (1.10\%, $0.36 \%$ to $2.96 \%)$.

\section{CONCLUSIONS}

Eighty one per cent of the countries of the world lack information on the epidemiology of psoriasis. The disease occurs more frequently in adults than in children. Psoriasis is unequally distributed across geographical regions; it is more frequent in high income countries and in regions with older populations. The estimates provided can help guide countries and the international community when making public health decisions on the appropriate management of psoriasis and assessing its natural history over time.

\section{SYSTEMATIC REVIEW REGISTRATION PROSPERO CRD42019160817.}

\section{Introduction}

Psoriasis is a chronic, immune mediated inflammatory skin disease, ${ }^{1}$ consisting of red, scaly plaques occurring most commonly on the elbows, knees, scalp, and lower back, but any skin surface can be involved. The condition greatly affects people's quality of life to the extent that it could be life ruining and stigmatising. ${ }^{2}$ Psoriasis is now considered a systemic disease; it is associated with psychological, metabolic, arthritic, and cardiovascular comorbidities. Lifespan is reduced as a consequence. ${ }^{3}$ In addition to the psychological and social burden related to psoriasis, the cost to patients and healthcare systems is high. ${ }^{4}$ Psoriasis can occur at any age, although most patients present with the condition before 35 years old. ${ }^{5}$ In 2014 the World Health Organization recognised psoriasis as a serious non-communicable disease ${ }^{6}$ and the accompanying WHO report $(2016)^{6}$ emphasised the need to better understand the global burden of the disease. To address this need, the Global Psoriasis Atlas (www.globalpsoriasisatlas.org) was established to conduct further research into the global prevalence and incidence of psoriasis, thereby helping to ensure better access to care for people with the disease.

Studies that report information on the incidence of psoriasis are limited. Current estimates come from Europe and North America, and mainly report on the incidence of psoriasis in adults or in the overall population. Additionally, several studies have reported on the prevalence of psoriasis. Earlier study specific estimates of the prevalence of psoriasis in adults range between $0.27 \%$ (95\% confidence interval 0.17 to $0.36)^{7}$ and $11.4 \%,{ }^{8}$ with age, sex, geography, ethnicity, genetic and environmental factors contributing to the variation in the prevalence of the disease. ${ }^{9}$ Higher prevalence rates have been reported at higher latitudes 
and in white people compared with other ethnic groups. ${ }^{10}$

The Global Burden of Disease group has generated estimates on the prevalence of psoriasis for 21 regions of the world, ${ }^{11}$ and more recently for the global population. ${ }^{12}$ These estimates relate to wider regions, ${ }^{11} 12$ but not to individual countries. Over the past decade there has been an increase in research examining the epidemiology of psoriasis, particularly in countries where estimates were lacking. Recent studies have often been derived from better data quality resources, many of them extracting information from large, routinely collected and electronic health databases, which are more nationally representative than previous studies.

Given the existing gaps in knowledge about the epidemiology of psoriasis, the aim of the study was to perform a systematic review and meta-analysis examining the incidence and prevalence of the disease. Additionally, the study aimed to generate global, regional, and country specific estimates of the prevalence of psoriasis.

\section{Methods}

The systematic review and statistical model follow the PRISMA (preferred reporting items for systematic reviews and meta-analyses) and GATHER (guidelines for accurate and transparent health estimates reporting) guidelines (supplementary material 4, eTables 12 and 13, respectively).

\section{Study design}

We conducted a systematic review of the incidence and prevalence of psoriasis that involved several steps. These steps included data identification and extraction; a descriptive summary of incidence data; and a statistical analysis to generate estimates of the global, regional, and country specific prevalence of psoriasis by using the information extracted from the included studies examining the prevalence of the disease.

\section{Data identification and extraction Search strategy}

We systematically searched 11 electronic and regional databases (Medline, Embase, Web of Science, SciELO, Korean Journal Databases, Russian Science Citation Index, WPRIM, SaudiMedLit, Informit, IndMed, and HERDIN) from their respective inception dates to October 2019. The main search terms were "psoriasis" ("psoriatic skin," "pustulosis"), "incidence” ("incident studies" or "cohort studies," or "longitudinal studies"), and "prevalence" ("prevalent studies" or "cross-sectional studies"). Supplementary material 1 gives full details about the search strategy. We also screened the references of all included studies and published review articles to identify any additional eligible studies.

\section{Inclusion and exclusion criteria}

We included studies if they reported on the prevalence or incidence of psoriasis in the general population. No language restrictions were applied. We also included studies that estimated the prevalence or incidence of other skin or autoimmune diseases, but provided data on psoriasis. Studies that used dermatology clinic case series, or specific subgroups of the population, or only focused on psoriatic arthritis were excluded.

\section{Data extraction}

In the first stage, two authors (RP and IYKI) independently screened the titles and abstracts identified from searching the databases for eligibility. Eligible papers were critically appraised and those meeting the inclusion criteria were selected for data extraction. We critically appraised all included studies for risk of bias by using the appraisal tool for crosssectional studies (AXIS). ${ }^{13}$ This quality assessment tool comprises 20 items that cover several domains: identification of research aim, appropriateness of study design, use of valid measures and statistical analyses, and consideration of bias. Supplementary material 1 (text 2.5) and supplementary material 3 (eTable 14) give full details about the AXIS tool, the items included, and the risk of bias assessment. Studies were classified as having high, medium, or low risk of bias, or were rated as unclear according to the quality of the methods used and results reported in the study. The two authors independently made these judgments, and reached consensus on the final rating if needed.

\section{Analysis of incidence data}

Because a limited number of studies reported on the incidence of psoriasis, these were summarised descriptively. We report incidence rates per 100000 person years (95\% confidence intervals). Results were analysed by country and age category (children, adults, or all ages), and if possible, we explored variation in the incidence of psoriasis within each country and age category.

\section{Statistical analysis of prevalence data}

After data extraction, we developed a filtering process for inclusion of studies in the statistical model because some studies used the same data resource. To prevent duplication, we included studies with the most recent or complete data on the variable of interest, or the most robust in terms of the methods used (supplementary material 1, eTable 2).

We fitted a bayesian hierarchical linear mixed model to estimate the global, regional, and country specific prevalence of psoriasis. This type of statistical model is considered the gold standard when data are sparse and heterogeneous. ${ }^{14}$ In the bayesian hierarchical model, estimates of the prevalence of psoriasis were informed by study data from the same country, if available, and by study data from other countries. We briefly describe the model below; supplementary material 2 (text 4) provides a full description.

The outcome was the log transformed prevalence of psoriasis, which allowed us to use a linear model and ensure predictions within the $0-100 \%$ range when back transformed. We mapped countries according 
to the Global Burden of Disease classification: 189 countries were nested in 21 regions, and regions were nested in seven super regions (table 1), the hierarchy of which mainly follows geography and income. High levels of heterogeneity in the global prevalence of psoriasis were expected for several reasons: varying age strata, or whether the prevalence was estimated in children, adults, or the overall population; type of diagnostic method, or whether the diagnosis was made by a physician, a dermatologist, or was self-reported; and type of estimate, or whether the estimate was calculated as point, period, or lifetime prevalence. ${ }^{8}$ Therefore, the hierarchical model had four levels (global, super regions, regions, and countries), four random intercepts for these, and three fixed covariates: age strata, type of diagnostic method (physician, dermatologist, or self-reported diagnosis), and type of prevalence measure (point, period, or lifetime prevalence). Age strata included children, adults, or the overall population (children and adults combined).

We used geographical clustering in the model to inform and generate estimates for countries with missing information. In particular, for countries with no data available, the model estimates were based on borrowed evidence from higher levels. For example, the region estimate value was used for the country estimate when no country specific data were available; the super region estimate value was used for countries when no data were available within a region. It is also noteworthy that the region and super region estimates are always obtained from studies identified at the country level; therefore there are no regional or super regional level studies. Supplementary material 2 (eTables 6-11) reports information on countries with observed or missing data.

The statistical model was fitted using bayesian inference, sampling from the posterior distribution over the parameters by using the Hamiltonian Markov chain Monte Carlo method. We used four chains of 2000 iterations each to run the model. After fitting the model, posterior predictions were made for each country and age strata permutation. We provide prevalence estimates in the context of $95 \%$ uncertainty intervals. To obtain the number of people affected by psoriasis by country, each country specific prevalence estimate was multiplied by the size of its population; we used the United Nations population structure for the year $2017 .{ }^{15}$ We assessed the fit of each model by evaluating the measures relative to the effective sample size and autocorrelation, and the trace plots (supplementary material 2, eFig 2). All analyses were performed in R (version 3.6.1) by using the RStanArm package, which relies on Stan.

\section{Patient and public involvement}

The International Federation of Psoriasis Associations (IFPA) are a collaborating organisation on the Global Psoriasis Atlas. Members of IFPA include people with psoriasis and our work plans and findings were presented during Global Psoriasis Atlas Steering Group meetings at which IFPA members asked questions about the study design and findings.

\section{Results}

We identified 41164 records by searching the databases. Of the 308 papers that were critically appraised and assessed for eligibility, 168 reported on the incidence or prevalence of psoriasis in the general population (supplementary material 1, eFig 1). Specifically, nine studies focused on the incidence of psoriasis (eTable 1), 145 reported on the prevalence of psoriasis (eTable 2), and 14 studies reported on both incidence and prevalence (eTable 1).

\section{Incidence of psoriasis}

The 23 studies that reported on the incidence of psoriasis in the general population were conducted mainly in western and eastern Europe (16 studies), and North America (six studies). Twelve studies reported on the incidence of psoriasis in all ages, with the incidence of the disease varying from 31.4 per 100000 person years in eastern Europe (Russia) ${ }^{16}$ to 521.1 per 100000 person years in western Europe (Germany). ${ }^{17}$ Springate and colleagues ${ }^{3}$ reported a slight decline in the incidence of psoriasis in all ages in the United Kingdom (from 159.0 to 129.0 per 100000 person years between 1999 and 2013). Kubanova and colleagues ${ }^{18}$ also reported a slight decline in the incidence in Russia (from 69.8 to 65.0 per 100000 person years between 2010 and 2016). However, the incidence of psoriasis in Denmark has been inconsistent, with a decrease from 140.1 to 104.0 per 100000 person years between 2003 and 2005, followed by an increase to 181.0 in 2010, which then decreased to 151.2 in 2012 (table 2). ${ }^{19}$

Only two studies, from Italy and the United States, explored the incidence of psoriasis in children over a seven year and 30 year period, respectively (table $2)^{20} 21$ While Tollefson and colleagues ${ }^{21}$ reported a steady rise in the incidence of psoriasis in the US between 1970 and 2000, Cantarutti and colleagues ${ }^{20}$ found that the incidence of psoriasis in Italy was stable between 2006 and 2012 (table 2).

The incidence of psoriasis was higher in adults than in children, varying from 30.3 per 100000 person years (95\% confidence interval 26.6 to 34.1$)^{22}$ in Taiwan to 321.0 per 100000 person years in Italy. ${ }^{23}$ While the data show a steadily increasing trend in incidence of psoriasis in adults in the US between 1970 and 2000 (table 2), ${ }^{24}$ data from Canada, Italy, and Taiwan show a slightly decreasing trend in incidence over time. ${ }^{22} 2325$

\section{Variation in the incidence of psoriasis by age and sex}

The incidence of psoriasis in children increased with age from 13.5 per 100000 person years (0-3 years old) to 53.1 per 100000 person years (14-18 years old; table 3). ${ }^{21}$ Despite higher estimates of psoriasis incidence from the UK ${ }^{26} 27$ than the US, ${ }^{2428}$ all of these studies showed a similar trend of increasing psoriasis incidence up to 39 years of age. The incidence then decreased at 40-49 years before increasing again with a second peak at around 50-59 years $\left(\mathrm{UK}^{27}\right)$ or $60-69$ years $\left(\mathrm{UK}^{26}\right.$ and $\mathrm{US}^{24}{ }^{28}$ ). The incidence of psoriasis decreased towards the end of life. 


\begin{tabular}{|c|c|}
\hline Region & Countries \\
\hline \multicolumn{2}{|c|}{ Central Europe, eastern Europe, and central Asia } \\
\hline Asia, central & Armenia, Azerbaijan, Georgia, Kazakhstan, Kyrgyzstan, Mongolia, Tajikistan, Turkmenistan, Uzbekistan \\
\hline Europe, central & $\begin{array}{l}\text { Albania, Bosnia and Herzegovina, Bulgaria, Croatia, Czech Republic, Hungary, Montenegro, Poland, Romania, Serbia, Slovakia, Slovenia, TFYR } \\
\text { Macedonia }\end{array}$ \\
\hline Europe, eastern & Belarus, Estonia, Latvia, Lithuania, Moldova, Russia, Ukraine \\
\hline \multicolumn{2}{|l|}{ High income } \\
\hline Asia Pacific, high income & Brunei Darussalam, Japan, Republic of Korea, Singapore \\
\hline Australasia & Australia, New Zealand \\
\hline Europe, western & $\begin{array}{l}\text { Austria, Belgium, Cyprus, Denmark, Finland, France, Germany, Greece, Iceland, Ireland, Israel, Italy, Luxembourg, Malta, Netherlands, Norway, } \\
\text { Portugal, Spain, Sweden, Switzerland, United Kingdom }\end{array}$ \\
\hline Latin America, southern & Argentina, Chile, Uruguay \\
\hline North America, high income & Canada, United States of America \\
\hline \multicolumn{2}{|l|}{ Latin America and Caribbean } \\
\hline Caribbean & $\begin{array}{l}\text { Antigua and Barbuda, Bahamas, Barbados, Belize, Cuba, Dominican Republic, Grenada, Guyana, Haiti, Jamaica, Puerto Rico, Saint Lucia, } \\
\text { Saint Vincent and the Grenadines, Suriname, Trinidad and Tobago, Virgin Island (US) }\end{array}$ \\
\hline Latin America, Andean & Bolivia, Ecuador, Peru \\
\hline Latin America, central & Colombia, Costa Rica, El Salvador, Guatemala, Honduras, Mexico, Nicaragua, Panama, Venezuela (Bolivarian Republic of) \\
\hline Latin America, tropical & Brazil, Paraguay \\
\hline \multicolumn{2}{|l|}{ North Africa and Middle East } \\
\hline North Africa and the Middle East & $\begin{array}{l}\text { Afghanistan, Algeria, Bahrain, Egypt, Iran (Islamic Republic of), Iraq, Jordan, Kuwait, Lebanon, Libyan Arab Jamahiriya, Morocco, Occupied } \\
\text { Palestinian Territory, Oman, Qatar, Saudi Arabia, Sudan, Syrian Arab Republic, Sudan, Tunisia, Turkey, United Arab Emirates, Yemen }\end{array}$ \\
\hline \multicolumn{2}{|l|}{ South Asia } \\
\hline Asia, south & Bangladesh, Bhutan, India, Nepal, Pakistan \\
\hline \multicolumn{2}{|c|}{ South East Asia, east Asia, and Oceania } \\
\hline Asia, east & China, Dem. People’s Republic of Korea, Taiwan \\
\hline Asia, South East & $\begin{array}{l}\text { Cambodia, Indonesia, Lao People’s Democratic Republic, Malaysia, Maldives, Mauritius, Myanmar, Philippines, Seychelles, Sri Lanka, } \\
\text { Thailand, Timor-Leste, Vietnam }\end{array}$ \\
\hline Oceania & Fiji, Guam, Kiribati, Marshall Islands, Micronesia (Fed. States of), Papua New Guinea, Samoa, Solomon Islands, Tonga, Vanuatu \\
\hline \multicolumn{2}{|l|}{ Sub-Saharan Africa } \\
\hline Sub-Saharan Africa, central & Angola, Central African Republic, Congo, Democratic Republic of the Congo, Equatorial Guinea, Gabon \\
\hline Sub-Saharan Africa, eastern & $\begin{array}{l}\text { Burundi, Comoros, Djibouti, Eritrea, Ethiopia, Kenya, Madagascar, Malawi, Mozambique, Rwanda, Somalia, South Sudan, Uganda, United } \\
\text { Republic of Tanzania, Zambia }\end{array}$ \\
\hline Sub-Saharan Africa, southern & Botswana, Lesotho, Namibia, South Africa, Swaziland, Zimbabwe \\
\hline Sub-Saharan Africa, western & $\begin{array}{l}\text { Benin, Burkina Faso, Côte d’Ivoire, Cameroon, Cape Verde, Chad, Gambia, Ghana, Guinea, Guinea-Bissau, Liberia, Mali, Mauritania, Niger, } \\
\text { Nigeria, São Tomé and Príncipe, Senegal, Sierra Leone, Togo }\end{array}$ \\
\hline
\end{tabular}

We found a lack of agreement in the published studies about variations in incidence rates by sex. Although the overall incidence rate was higher in girls than in boys aged less than 18 years (43.9 and 37.9 per 100000 person years, respectively), this pattern was not consistent across all age bands. ${ }^{21}$
Some studies reported higher incidence in women than in men, ${ }^{3192628}$ whereas other studies presented contrasting results. ${ }^{23} 24$ When we examined the incidence of psoriasis by sex and age bands, the two peaks for age at onset in women were more frequently around 18-29 and 50-59 years, whereas in men they

\begin{tabular}{|c|c|c|c|c|c|c|}
\hline \multirow{2}{*}{$\begin{array}{l}\text { Study, country, study } \\
\text { period }\end{array}$} & \multirow{2}{*}{ Diagnostic method } & \multirow{2}{*}{$\begin{array}{l}\text { Age of } \\
\text { population }\end{array}$} & \multirow{2}{*}{$\begin{array}{l}\text { No with } \\
\text { psoriasis }\end{array}$} & \multicolumn{3}{|c|}{ Incidence rate per 100000 person years $(95 \% \mathrm{Cl})$} \\
\hline & & & & Entire group & Female population & Male population \\
\hline \multicolumn{7}{|c|}{ Children } \\
\hline \multicolumn{7}{|c|}{ Cantarutti et al (2015), Italy } \\
\hline $2006-12$ & Family paediatrician & $\leq 14$ & - & - & - & - \\
\hline 2006 & & & & $61.0(50.0 \text { to } 80.0)^{\star}$ & - & - \\
\hline 2007 & & & & $45.0(30.0 \text { to } 60.0)^{*}$ & - & - \\
\hline 2008 & & & & $54.0(40.0 \text { to } 70.0)^{\star}$ & - & - \\
\hline 2009 & & & & $53.0(40.0 \text { to } 70.0)^{\star}$ & - & - \\
\hline 2010 & & & & $53.0(40.0 \text { to } 70.0)^{*}$ & - & - \\
\hline 2011 & & & & $40.0(30.0 \text { to } 50.0)^{*}$ & - & - \\
\hline 2012 & & & & $57.0(40.0 \text { to } 80.0)^{*}$ & - & - \\
\hline \multicolumn{7}{|c|}{ Tollefson et al (2010), US } \\
\hline $1970-99$ & Dermatologist or physician & $<18$ & 357 & $40.8(36.6 \text { to } 45.1)^{*} \dagger$ & $43.9(37.6 \text { to } 50.2)^{*} \dagger$ & $37.9(32.2 \text { to } 43.6)^{\star}+$ \\
\hline $1970-74$ & & & & $29.6(20.9 \text { to } 38.3)^{*} \dagger$ & - & - \\
\hline $1975-79$ & & & & $35.7(25.9 \text { to } 45.5)^{*} \dagger$ & - & - \\
\hline $1980-84$ & & & & $31.4(22.0 \text { to } 40.8)^{\star} \dagger$ & - & - \\
\hline 1985-89 & & & & $42.7(31.8 \text { to } 53.7)^{\star} \dagger$ & - & - \\
\hline 1990-94 & & & & $40.0(29.7 \text { to } 50.3)^{\star} \dagger$ & - & - \\
\hline 1995-99 & & & & $62.7(50.4 \text { to } 65.0)^{*} \dagger$ & - & - \\
\hline
\end{tabular}




\begin{tabular}{|c|c|c|c|c|c|c|}
\hline \multirow{2}{*}{$\begin{array}{l}\text { Study, country, study } \\
\text { period }\end{array}$} & \multirow{2}{*}{ Diagnostic method } & \multirow{2}{*}{$\begin{array}{l}\text { Age of } \\
\text { population }\end{array}$} & \multirow{2}{*}{$\begin{array}{l}\text { No with } \\
\text { psoriasis }\end{array}$} & \multicolumn{3}{|c|}{ Incidence rate per 100000 person years $(95 \% \mathrm{Cl})$} \\
\hline & & & & Entire group & Female population & Male population \\
\hline \multicolumn{7}{|c|}{ Adults } \\
\hline \multicolumn{7}{|c|}{ Eder et al (2017), Canada } \\
\hline $2000-15$ & Physician & $\geq 20$ & \multirow{17}{*}{-} & - & - & - \\
\hline 2000 & & & & $114.0(112.0 \text { to } 116.0)^{\star} \dagger$ & - & - \\
\hline 2001 & & & & $111.0(109.0 \text { to } 113.0)^{\star} \dagger$ & - & - \\
\hline 2002 & & & & $103.0(101.0 \text { to } 105.0)^{\star} \dagger$ & - & - \\
\hline 2003 & & & & $101.0(99.0 \text { to } 103.0)^{\star} \dagger$ & - & - \\
\hline 2004 & & & & $101.0(99.0 \text { to } 103.0)^{*} \dagger$ & - & - \\
\hline 2005 & & & & $97.0(95.0 \text { to } 99.0)^{*} \dagger$ & - & - \\
\hline 2006 & & & & $97.0(95.0 \text { to } 99.0)^{\star} \dagger$ & - & - \\
\hline 2007 & & & & $96.0(94.0 \text { to } 98.0)^{*} \dagger$ & - & - \\
\hline 2008 & & & & $96.0(94.0 \text { to } 98.0)^{*} \dagger$ & - & - \\
\hline 2009 & & & & $95.0(93.0 \text { to } 97.0)^{*} \dagger$ & - & - \\
\hline 2010 & & & & $95.0(93.0 \text { to } 97.0)^{\star} \dagger$ & - & - \\
\hline 2011 & & & & $98.0(96.0 \text { to } 100.0)^{\star}+$ & - & - \\
\hline 2012 & & & & $100.0(98.0 \text { to } 102.0)^{\star} \dagger$ & - & - \\
\hline 2013 & & & & $105.0(103.0 \text { to } 107.0)^{\star} \dagger$ & - & - \\
\hline 2014 & & & & $102.0(100.0 \text { to } 104.0)^{\star} \dagger$ & - & - \\
\hline 2015 & & & & $105.0(103.0 \text { to } 107.0)^{\star} \dagger$ & - & - \\
\hline \multicolumn{7}{|c|}{ Eder et al (2019), Canada } \\
\hline $2000-15$ & \multirow[t]{17}{*}{ Physician } & \multirow[t]{17}{*}{$\geq 20$} & - & - & - & - \\
\hline 2000 & & & 9873 & $111.1(108.9 \text { to } 113.4)^{\star} \dagger$ & - & - \\
\hline 2001 & & & 9849 & $108.6(106.5 \text { to } 110.8)^{\star} \dagger$ & - & - \\
\hline 2002 & & & 9203 & $99.7(97.6 \text { to } 101.8)^{\star} \dagger$ & - & - \\
\hline 2003 & & & 9111 & $97.3(95.3 \text { to } 99.3)^{*} \dagger$ & - & - \\
\hline 2004 & & & 9060 & $95.3(93.3 \text { to } 97.3)^{\star} \dagger$ & - & - \\
\hline 2005 & & & 8645 & $89.8(87.9 \text { to } 91.8)^{*} \dagger$ & - & - \\
\hline 2006 & & & 8979 & $92.7(90.7 \text { to } 94.7)^{\star} \dagger$ & - & - \\
\hline 2007 & & & 8601 & $87.6(85.7 \text { to } 89.6)^{\star} \dagger$ & - & - \\
\hline 2008 & & & 8585 & $85.9(84.0 \text { to } 87.8)^{\star} \dagger$ & - & - \\
\hline 2009 & & & 8610 & $85.2(83.4 \text { to } 87.1)^{\star} \dagger$ & - & - \\
\hline 2010 & & & 8399 & $81.8(80.0 \text { to } 83.7)^{\star} \dagger$ & - & - \\
\hline 2011 & & & 8868 & $85.0(83.2 \text { to } 86.9)^{\star} \dagger$ & - & - \\
\hline 2012 & & & 8883 & $83.9(82.1 \text { to } 85.7)^{\star} \dagger$ & - & - \\
\hline 2013 & & & 8789 & $82.1(80.3 \text { to } 83.9)^{\star} \dagger$ & - & - \\
\hline 2014 & & & 8212 & $76.1(74.4 \text { to } 77.9)^{\star} \dagger$ & - & - \\
\hline 2015 & & & 7541 & $68.7(67.1 \text { to } 70.3)^{\star} \dagger$ & - & - \\
\hline \multicolumn{7}{|c|}{ Vena et al (2010), Italy } \\
\hline $2001-05$ & \multirow[t]{3}{*}{ Physician } & $\geq 18$ & 5792 & - & - & - \\
\hline 2001 & & & & $321.0^{*}$ & $291.0^{*}$ & $357.0^{*}$ \\
\hline 2005 & & & & $230.0^{*}$ & $207.0^{*}$ & $254.0^{*}$ \\
\hline Pezzolo et al (2019), It & & & & & & \\
\hline $2003-04$ & Self-reported diagnosis & $\geq 25$ & - & $302(232 \text { to } 392)^{\star}$ & $380(270 \text { to } 535)^{\star}$ & $296(197 \text { to } 443)^{\star}$ \\
\hline Wei et al (2018), Taiwa & & & & & & \\
\hline 2001 & - & $\geq 16$ & 333 & $42.0(37.5 \text { to } 46.5)^{\star} \dagger$ & - & - \\
\hline 2002 & & & 320 & $40.1(35.7 \text { to } 44.5)^{\star} \dagger$ & - & - \\
\hline 2003 & & & 329 & $41.7(37.2 \text { to } 46.3)^{\star} \dagger$ & - & - \\
\hline 2004 & & & 370 & $46.9(42.1 \text { to } 51.8)^{\star} \dagger$ & - & - \\
\hline 2005 & & & 272 & $33.8(29.7 \text { to } 37.8)^{\star} \dagger$ & - & - \\
\hline 2006 & & & 325 & $39.7(35.3 \text { to } 44.1)^{\star} \dagger$ & - & - \\
\hline 2007 & & & 295 & $35.7(31.5 \text { to } 39.8)^{\star} \dagger$ & - & - \\
\hline 2008 & & & 291 & $34.1(30.1 \text { to } 38.2)^{\star} \dagger$ & - & - \\
\hline 2009 & & & 322 & $37.8(33.5 \text { to } 42.0)^{\star} \dagger$ & - & - \\
\hline 2010 & & & 316 & $36.7(32.5 \text { to } 40.9)^{\star} \dagger$ & - & - \\
\hline 2011 & & & 338 & $40.3(35.8 \text { to } 44.8)^{\star} \dagger$ & - & - \\
\hline 2012 & & & 327 & $37.2(33.0 \text { to } 41.5)^{\star} \dagger$ & - & - \\
\hline 2013 & & & 273 & $30.3(26.6 \text { to } 34.1)^{\star} \dagger$ & - & - \\
\hline Khalid et al (2013), UK & & & & & & \\
\hline $2007-09$ & Physician & $\geq 18$ & 10832 & $280.0(280.0 \text { to } 290.0)^{\star} \dagger$ & - & - \\
\hline Tillett et al (2017), UK & & & & & & \\
\hline $1998-2014$ & Physician & $\geq 18$ & 88858 & $183.0(182.0 \text { to } 184.0)^{\star}$ & $186.0(184.0 \text { to } 188.0)^{*}$ & $179.0(178.0 \text { to } 181.0)^{\star}$ \\
\hline Shbeeb et al (1995), U & & & & & & \\
\hline $1982-91$ & - & - & - & $78.0(70.0 \text { to } 86.0)^{\star} \dagger$ & - & - \\
\hline
\end{tabular}




\begin{tabular}{|c|c|c|c|c|c|c|}
\hline \multicolumn{7}{|l|}{ Table 2 | Continued } \\
\hline \multirow{2}{*}{$\begin{array}{l}\text { Study, country, study } \\
\text { period }\end{array}$} & \multirow{2}{*}{ Diagnostic method } & \multirow{2}{*}{$\begin{array}{l}\text { Age of } \\
\text { population }\end{array}$} & \multirow{2}{*}{$\begin{array}{l}\text { No with } \\
\text { psoriasis }\end{array}$} & \multicolumn{3}{|c|}{ Incidence rate per 100000 person years $(95 \% \mathrm{Cl})$} \\
\hline & & & & Entire group & Female population & Male population \\
\hline \multicolumn{7}{|l|}{ Icen et al (2009), US } \\
\hline $1970-2000$ & \multirow[t]{7}{*}{ Dermatologist or physician } & \multirow[t]{7}{*}{$\geq 18$} & \multirow[t]{7}{*}{1633} & $78.9(75.0 \text { to } 82.9)^{*}+\ddagger$ & $73.2(68.0 \text { to } 78.4)^{*}+\neq$ & $85.5(79.5 \text { to } 91.6)^{\star}+\ddagger$ \\
\hline $1970-74$ & & & & $50.8(41.9 \text { to } 59.6)^{\star}+\ddagger$ & - & - \\
\hline $1975-79$ & & & & $53.2(44.8 \text { to } 61.6)^{\star}+\ddagger$ & - & - \\
\hline $1980-84$ & & & & $80.9(70.8 \text { to } 91.1)^{\star}+\ddagger$ & - & - \\
\hline $1985-89$ & & & & $78.9(69.5 \text { to } 88.4)^{\star}+\ddagger$ & - & - \\
\hline $1990-94$ & & & & $88.7(79.1 \text { to } 98.3)^{\star}+\ddagger$ & - & - \\
\hline 1995-99 & & & & $100.5(90.8 \text { to } 110.2)^{\star}+\ddagger$ & - & - \\
\hline \multicolumn{7}{|c|}{ All ages } \\
\hline \multicolumn{7}{|c|}{ Egeberg et al (2017), Denmark } \\
\hline $2003-12$ & Physician & $0-70+$ & - & - & - & - \\
\hline 2003 & & & & $140.1(137.1 \text { to } 143.2)^{*}$ & $146.8^{*}$ & $133.4^{\star}$ \\
\hline 2004 & & & & $122.2(119.4 \text { to } 125.1)^{\star}$ & $130.7^{\star}$ & $113.6^{\star}$ \\
\hline 2005 & & & & $104.0(101.4 \text { to } 106.7)^{\star}$ & $107.5^{\star}$ & $100.5^{\star}$ \\
\hline 2006 & & & & $105.5(102.9 \text { to } 108.2)^{\star}$ & $110.4^{*}$ & $100.4^{*}$ \\
\hline 2007 & & & & $111.5(108.7 \text { to } 114.2)^{\star}$ & $110.8^{*}$ & $112.2^{\star}$ \\
\hline 2008 & & & & $128.6(125.7 \text { to } 131.6)^{\star}$ & $128.8^{*}$ & $128.4^{*}$ \\
\hline 2009 & & & & $174.8(171.4 \text { to } 178.3)^{\star}$ & $192.6^{\star}$ & $156.8^{\star}$ \\
\hline 2010 & & & & $181.0(177.5 \text { to } 184.5)^{\star}$ & $199.5^{\star}$ & $162.3^{\star}$ \\
\hline 2011 & & & & $171.3(167.9 \text { to } 174.7)^{\star}$ & $187.9^{\star}$ & $154.5^{\star}$ \\
\hline 2012 & & & & $151.2(148.0 \text { to } 154.5)^{*}$ & $165.9^{\star}$ & $136.4^{\star}$ \\
\hline Jacob et al (2016), Gerr & many & & & & & \\
\hline $2007-10$ & Physician & - & 14686 & $521.1^{\star}$ & - & - \\
\hline Sewerin et al (2019), G & ermany & & & & & \\
\hline 2009 & - & - & - & - & 46.3 to $58.2^{*}$ & 35.4 to $50.3^{*}$ \\
\hline 2010 & & & & & 35.3 to $45.6^{*}$ & 26.4 to $39.4^{*}$ \\
\hline 2011 & & & & & 21.7 to $30.5^{*}$ & 17.3 to $29.3^{\star}$ \\
\hline 2012 & & & & & 19.1 to $26.4^{*}$ & 17.1 to $26.3^{*}$ \\
\hline Shalom et al (2018), Is & rael & & & & & \\
\hline 2016 & - & - & - & $246(241 \text { to } 251)^{*} \dagger$ & - & - \\
\hline 2017 & & & & $243(239 \text { to } 248)^{\star}+$ & - & - \\
\hline Schonmann et al (2019 & 9), Israel & & & & & \\
\hline 2011 & Dermatologist & $0-85+$ & 9770 & $282(276 \text { to } 288)^{\star} \dagger$ & $268(261 \text { to } 276)^{\star}+$ & $296(287 \text { to } 305)^{*}+$ \\
\hline 2012 & & & 9796 & $278(272 \text { to } 284)^{\star} \dagger$ & $273(265 \text { to } 281)^{\star}+$ & $283(275 \text { to } 292)^{*}+$ \\
\hline 2013 & & & 10430 & $291(285 \text { to } 297)^{\star} \dagger$ & $278(270 \text { to } 286)^{\star}+$ & $304(295 \text { to } 312)^{*}+$ \\
\hline 2014 & & & 10072 & $276(271 \text { to } 282)^{\star} \dagger$ & $263(256 \text { to } 271)^{\star \star}$ & $290(281 \text { to } 298)^{\star} \dagger$ \\
\hline 2015 & & & 10033 & $273(267 \text { to } 279)^{\star}+$ & $257(250 \text { to } 265)^{\star}+$ & $289(280 \text { to } 297)^{\star}+$ \\
\hline 2016 & & & 10505 & $281(276 \text { to } 287)^{\star} \dagger$ & $269(261 \text { to } 276)^{\star} \dagger$ & $294(286 \text { to } 302)^{\star} \dagger$ \\
\hline 2017 & & & 10489 & $276(270 \text { to } 281)^{\star} \dagger$ & $263(256 \text { to } 271)^{\star} \dagger$ & $288(280 \text { to } 296)^{\star} \dagger$ \\
\hline Znamenskaya et al (20 & 12), Russia & & & & & \\
\hline 2009 & Physician & $0-18+$ & 99988 & $70.5^{*}$ & - & - \\
\hline 2010 & & & 99348 & $69.8^{*}$ & - & - \\
\hline 2011 & & & 99436 & $69.6^{\star}$ & - & - \\
\hline Kubanova et al (2017), & Russia & & & & & \\
\hline 2010 & Physician & $0-18+$ & - & $69.8^{*}$ & - & - \\
\hline 2011 & & & & $69.6^{*}$ & - & - \\
\hline 2012 & & & & $68.4^{*}$ & - & - \\
\hline 2013 & & & & $65.9^{*}$ & $\begin{array}{llll}- & \\
\end{array}$ & $\begin{array}{lll}- & & \end{array}$ \\
\hline 2014 & & & & $64.7^{\star}$ & - & - \\
\hline 2015 & & & & $62.8^{*}$ & - & - \\
\hline 2016 & & & & $65.0^{*}$ & - & - \\
\hline Odinets et al (2017), Rs & ussia & & & & & \\
\hline 2010 & Physician & $0-18+$ & 1180 & $42.5^{*}$ & - & - \\
\hline 2011 & & & 1136 & $40.8^{\star}$ & - & - \\
\hline 2012 & & & 1257 & $45.1^{*}$ & - & - \\
\hline 2013 & & & 875 & $31.4^{\star}$ & - & - \\
\hline 2014 & & & 945 & $33.8^{\star}$ & - & - \\
\hline 2015 & & & 941 & $33.6^{\star}$ & - & - \\
\hline 2016 & & & 1094 & $39.0^{*}$ & - & - \\
\hline Donker et al (1998), $\mathrm{Ne}$ & etherlands & & & & & \\
\hline $1987-88$ & Physician & $0-65+$ & 106 & $130.0(120.0 \text { to } 140.0)^{*} \dagger$ & - & - \\
\hline Donker et al (1998), $\mathrm{Ne}$ & etherlands & & & & & \\
\hline 1995 & Physician & $0-65+$ & 24 & $120.0(70.0 \text { to } 190.0)^{*} \dagger$ & - & - \\
\hline Huerta et al (2007), UK & & & & & & \\
\hline $1996-97$ & Physician & $0-80+$ & 3994 & $140.0^{*}$ & - & - \\
\hline
\end{tabular}




\begin{tabular}{|c|c|c|c|c|c|c|}
\hline \multirow{2}{*}{$\begin{array}{l}\text { Study, country, study } \\
\text { period }\end{array}$} & \multirow{2}{*}{ Diagnostic method } & \multirow{2}{*}{$\begin{array}{l}\text { Age of } \\
\text { population }\end{array}$} & \multirow{2}{*}{$\begin{array}{l}\text { No with } \\
\text { psoriasis }\end{array}$} & \multicolumn{3}{|c|}{ Incidence rate per 100000 person years $(95 \% \mathrm{Cl})$} \\
\hline & & & & Entire group & Female population & Male population \\
\hline \multicolumn{7}{|c|}{ Springate et al (2017), UK } \\
\hline $1999-2013$ & \multirow[t]{16}{*}{ Physician } & \multirow[t]{16}{*}{$0-100$} & - & - & - & - \\
\hline 1999 & & & 4279 & $159.0(155.0 \text { to } 164.0)^{*}+$ & $161.0(155.0 \text { to } 168.0)^{\star} \dagger$ & $158.0(151.0 \text { to } 165.0)^{\star}+$ \\
\hline 2000 & & & 5398 & $163.0(158.0 \text { to } 167.0)^{\star} \dagger$ & $162.0(156.0 \text { to } 169.0)^{\star} \dagger$ & $163.0(157.0 \text { to } 170.0)^{\star}+$ \\
\hline 2001 & & & 6286 & $164.0(160.0 \text { to } 168.0)^{\star}+$ & $163.0(157.0 \text { to } 168.0)^{\star}+$ & $166.0(160.0 \text { to } 172.0)^{\star}+$ \\
\hline 2002 & & & 7259 & $170.0(166.0 \text { to } 174.0)^{*}+$ & $174.0(169.0 \text { to } 180.0)^{\star} \dagger$ & $166.0(161.0 \text { to } 172.0)^{\star}+$ \\
\hline 2003 & & & 7977 & $172.0(168.0 \text { to } 176.0)^{\star} \dagger$ & $178.0(173.0 \text { to } 183.0)^{\star} \dagger$ & $166.0(161.0 \text { to } 172.0)^{\star} \dagger$ \\
\hline 2004 & & & 8209 & $166.0(163.0 \text { to } 170.0)^{*} \dagger$ & $170.0(165.0 \text { to } 175.0)^{\star} \dagger$ & $163.0(158.0 \text { to } 168.0)^{\star}+$ \\
\hline 2005 & & & 8522 & $165.0(162.0 \text { to } 169.0)^{*}+$ & $173.0(168.0 \text { to } 178.0)^{\star} \dagger$ & $158.0(153.0 \text { to } 163.0)^{\star}+$ \\
\hline 2006 & & & 8499 & $161.0(158.0 \text { to } 165.0)^{\star}+$ & $169.0(164.0 \text { to } 174.0)^{\star}+$ & $154.0(149.0 \text { to } 159.0)^{\star}+$ \\
\hline 2007 & & & 8807 & $165.0(162.0 \text { to } 168.0)^{*} \dagger$ & $170.0(165.0 \text { to } 175.0)^{\star} \dagger$ & $160.0(155.0 \text { to } 165.0)^{\star}+$ \\
\hline 2008 & & & 8964 & $163.0(160.0 \text { to } 167.0)^{*}+$ & $165.0(160.0 \text { to } 170.0)^{\star} \dagger$ & $162.0(157.0 \text { to } 167.0)^{\star}+$ \\
\hline 2009 & & & 8518 & $155.0(152.0 \text { to } 158.0)^{\star}+$ & $159.0(154.0 \text { to } 163.0)^{\star}+$ & $152.0(147.0 \text { to } 156.0)^{\star}+$ \\
\hline 2010 & & & 7715 & $143.0(140.0 \text { to } 146.0)^{\star}+$ & $145.0(141.0 \text { to } 150.0)^{\star} \dagger$ & $140.0(136.0 \text { to } 145.0)^{\star}+$ \\
\hline 2011 & & & 7499 & $140.0(137.0 \text { to } 143.0)^{\star} \dagger$ & $144.0(140.0 \text { to } 149.0)^{\star} \dagger$ & $135.0(131.0 \text { to } 140.0)^{\star}+$ \\
\hline 2012 & & & 6992 & $131.0(128.0 \text { to } 134.0)^{*}+$ & $136.0(132.0 \text { to } 141.0)^{\star} \dagger$ & $126.0(122.0 \text { to } 130.0)^{\star}+$ \\
\hline 2013 & & & 6350 & $129.0(126.0 \text { to } 133.0)^{*}+$ & $131.0(127.0 \text { to } 136.0)^{\star}+$ & $127.0(123.0 \text { to } 132.0)^{\star}+$ \\
\hline \multicolumn{7}{|l|}{ Bell et al (1991), US } \\
\hline $1980-83$ & Dermatologist or physician & $0-70+$ & 132 & $59.9(49.5 \text { to } 70.3)^{*}+$ & $63.6(48.9 \text { to } 78.3)^{\star} \dagger$ & $58.4(42.8 \text { to } 74.1)^{*} \dagger$ \\
\hline
\end{tabular}

occurred more frequently around $30-39$ and $60-69$ or 70-79 years. $^{2426-28}$

\section{Prevalence of psoriasis}

The 159 studies that reported on the prevalence of psoriasis in the general population were identified from 12 regions of the world (table 4). Most studies (107; 67\%) were conducted in high income countries; these also contributed the highest number of nationally representative studies. Because of overlapping data sources, we included 129 independent data points in the statistical analysis; these came from 35 out of 189 (19\%) countries of the world (fig 1). Most studies included in the statistical analyses were graded as low-medium risk of bias ( $76 \%$; supplementary material 3, eTable 14). Supplementary material 2 (eTables 6-11) reports estimates of lifetime prevalence of psoriasis for the main analyses for each age stratum according to physician or dermatologist diagnosis, and self-reported lifetime prevalence; however, the number of people affected by psoriasis is reported for the adult population only.

Regionally, the occurrence of the disease for the overall population varied from $0.11 \%$ (95\% uncertainty interval $0.04 \%$ to $0.30 \%)$ in east Asia to $1.58 \%(0.50 \%$ to $5.73 \%$ ) in Australasia, and $1.52 \%$ (0.87\% to $2.74 \%$ ) in western Europe (fig 2). Country specific prevalence of psoriasis varied substantially. Considering the estimate for the overall population, Australia (1.88\%, 0.59\% to $6.10 \%)$, Norway $(1.86 \%, 0.94 \%$ to $3.97 \%)$, Israel (1.81\%, $0.83 \%$ to $4.44 \%)$, and Denmark $(1.79 \%, 0.91 \%$ to $3.61 \%$ ) had the highest estimates of the prevalence of psoriasis (supplementary material 2, eTable 8). The estimated prevalence of psoriasis in countries from east Asia was much lower, Taiwan being the country with the lowest prevalence worldwide (0.05\%; $0.02 \%$ to $0.16 \%)$.

Psoriasis occurred more frequently in adults than in children. The prevalence of psoriasis in children varied from $0.02 \%$ (95\% uncertainty interval $0.01 \%$ to $0.04 \%)$ in east Asia to $0.22 \%(0.06 \%$ to $0.81 \%)$ in Australasia and $0.21 \%(0.11 \%$ to $0.41 \%)$ in western Europe (fig 3). In adults, the disease varied from $0.14 \%(0.05 \%$ to $0.40 \%)$ in east Asia to $1.99 \%$ (0.64\% to $6.60 \%)$ in Australasia. Other regions with an occurrence of the disease above $1 \%$ were western Europe $(1.92 \%, 1.07 \%$ to $3.46 \%)$, central Europe (1.83\%, 0.62\% to 5.32\%), high income North America $(1.50 \%, 0.63 \%$ to $3.60 \%)$, and high income southern Latin America (1.10\%, 0.36\% to 2.96\%; fig 4).

Given the strong association of psoriasis with age, the prevalence of psoriasis also varied across countries because of differences in regional and country specific age structures. Considering country specific estimates, the physician diagnosed lifetime prevalence of psoriasis in adults was highest in Australia (2.38\%, $0.78 \%$ to $7.01 \%)$, Norway $(2.36 \%, 1.10 \%$ to $5.04 \%$ ), Israel $(2.28 \%, 0.98 \%$ to $5.65 \%)$, Denmark $(2.26 \%$, $1.15 \%$ to $4.58 \%$ ), Romania $(2.24 \%, 0.76 \%$ to $6.73 \%)$, Germany $(2.20 \%, 1.12 \%$ to $4.38 \%)$, Sweden $(2.10 \%$, $0.94 \%$ to $4.74 \%)$, Poland $(2.06 \%, 0.61 \%$ to $6.58 \%$ ), and Italy $(2.00 \%, 1.00 \%$ to $4.14 \%$; fig 5 and eTable 7 , supplementary material 2). However, the countries with the highest number of adults affected were the US (3.4 million, 95\% uncertainty interval 1.5 to 7.7 million), India (2.9 million, 0.8 to 10.0 million), China (2.3 million, 0.9 to 6.1 million), Germany (1.5 million, 0.8 to 2.9 million), Brazil (1.2 million, 0.3 to 4.8 million), France (1.0 million, 0.5 to 2.1 million), and the UK (1.0 million, 0.5 to 1.9). Globally, in 2017, an estimated 29.5 million adults had psoriasis, corresponding to a physician diagnosed lifetime prevalence of $0.59 \%$ (95\% uncertainty interval 0.19\% to $1.66 \%$ ) of the adult population worldwide.

Additional sources of heterogeneity in the estimates were factors such as type of diagnostic method 


\begin{tabular}{|c|c|c|c|c|c|c|}
\hline \multirow{2}{*}{$\begin{array}{l}\text { Study, country, and } \\
\text { study period }\end{array}$} & \multirow{2}{*}{ Diagnostic method } & \multirow{2}{*}{$\begin{array}{l}\text { Age of } \\
\text { population }\end{array}$} & \multirow{2}{*}{$\begin{array}{l}\text { No with } \\
\text { psoriasis }\end{array}$} & \multicolumn{3}{|c|}{ Incidence rate per 100000 person years $(95 \% \mathrm{Cl})$} \\
\hline & & & & Entire group & Female population & Male population \\
\hline \multicolumn{7}{|l|}{ Children } \\
\hline \multicolumn{7}{|c|}{ Tollefson et al (2010), US } \\
\hline \multirow[t]{6}{*}{$1970-99$} & \multirow{6}{*}{$\begin{array}{l}\text { Dermatologist or } \\
\text { physician }\end{array}$} & $<18$ & 357 & $40.8(36.6 \text { to } 45.1)^{\star} \dagger$ & $43.9(37.6 \text { to } 50.2)^{\star} \dagger$ & $37.9(32.2 \text { to } 43.6)^{*} \dagger$ \\
\hline & & $0-3$ & 27 & $13.5^{\star}$ & $13.2^{*}$ & $13.7^{\star}$ \\
\hline & & 4-7 & 84 & $42.2^{*}$ & $40.2^{*}$ & $44.1^{*}$ \\
\hline & & 8-10 & 69 & $44.0^{*}$ & $55.7^{\star}$ & $33.2^{*}$ \\
\hline & & 11-13 & 75 & $52.2^{*}$ & $49.6^{*}$ & $54.6^{*}$ \\
\hline & & $14-17$ & 102 & $53.1^{*}$ & $61.9^{*}$ & $44.7^{\star}$ \\
\hline \multicolumn{7}{|l|}{ Adults } \\
\hline \multicolumn{7}{|c|}{ Pezzolo et al (2019), Italy } \\
\hline \multirow[t]{7}{*}{$2003-04$} & \multirow[t]{7}{*}{ Self-reported diagnosis } & $\geq 25$ & - & $302(232 \text { to } 392)^{\star}$ & $380(270 \text { to } 535)^{\star}$ & $296(197 \text { to } 443)^{\star}$ \\
\hline & & $<35$ & - & $186(95 \text { to } 365)^{\star}$ & $236(101 \text { to } 554)^{\star}$ & $137(45 \text { to } 412)^{\star}$ \\
\hline & & $35-44$ & - & $342(193 \text { to } 605)^{*}$ & $251(98 \text { to } 642)^{\star}$ & $433(211 \text { to } 889)^{\star}$ \\
\hline & & $45-54$ & - & $219(110 \text { to } 435)^{\star}$ & $339(156 \text { to } 739)^{\star}$ & $96(22 \text { to } 419)^{\star}$ \\
\hline & & $55-64$ & - & $385(223 \text { to } 665)^{\star}$ & $492(252 \text { to } 961)^{\star}$ & $269(105 \text { to } 690)^{\star}$ \\
\hline & & $65-74$ & - & $420(253 \text { to } 697)^{\star}$ & $395(199 \text { to } 782)^{\star}$ & $456(214 \text { to } 969)^{\star}$ \\
\hline & & $\geq 74$ & - & - & - & - \\
\hline \multicolumn{7}{|l|}{ Khalid et al (2013), UK } \\
\hline \multirow[t]{8}{*}{$2007-09$} & \multirow[t]{8}{*}{ Physician } & $\geq 18$ & 10832 & $280.0(280.0 \text { to } 290.0)^{\star} \dagger$ & - & - \\
\hline & & $18-29$ & & - & $350.0(320.0 \text { to } 380.0)^{\star}$ & $250.0(230.0 \text { to } 280.0)^{\star}$ \\
\hline & & $30-39$ & & - & $320.0(280.0 \text { to } 350.0)^{\star}$ & $290.0(260.0 \text { to } 320.0)^{*}$ \\
\hline & & $40-49$ & & - & $220.0(200.0 \text { to } 240.0)^{\star}$ & $220.0(200.0 \text { to } 250.0)^{*}$ \\
\hline & & $50-59$ & & - & $310.0(280.0 \text { to } 340.0)^{\star}$ & $320.0(300.0 \text { to } 360.0)^{\star}$ \\
\hline & & $60-69$ & & - & $310.0(280.0 \text { to } 350.0)^{\star}$ & $370.0(330.0 \text { to } 400.0)^{\star}$ \\
\hline & & $70-79$ & & - & $290.0(250.0 \text { to } 320.0)^{*}$ & $290.0(250.0 \text { to } 330.0)^{\star}$ \\
\hline & & $\geq 80$ & & - & $160.0(130.0 \text { to } 190.0)^{\star}$ & $180.0(140.0 \text { to } 230.0)^{\star}$ \\
\hline Tillett et al (2017), UK & & & & & & \\
\hline $1998-2014$ & Physician & 218 & 88858 & $183.0(182.0 \text { to } 184.0)^{\star}$ & $186.0(184.0 \text { to } 188.0)^{\star}$ & $179.0(178.0 \text { to } 181.0)^{\star}$ \\
\hline & & $18-29$ & 14292 & $174.0(171.0 \text { to } 177.0)^{\star}$ & $193.0(188.0 \text { to } 197.0)^{\star}$ & $157.0(154.0 \text { to } 161.0)^{\star}$ \\
\hline & & $30-39$ & 15169 & $176.0(173.0 \text { to } 179.0)^{\star}$ & $182.0(178.0 \text { to } 186.0)^{\star}$ & $170.0(166.0 \text { to } 173.0)^{\star}$ \\
\hline & & $40-49$ & 15423 & $164.0(162.0 \text { to } 167.0)^{\star}$ & $158.0(155.0 \text { to } 162.0)^{\star}$ & $170.0(166.0 \text { to } 174.0)^{\star}$ \\
\hline & & $50-59$ & 16218 & $195.0(192.0 \text { to } 198.0)^{*}$ & $200.0(196.0 \text { to } 204.0)^{\star}$ & $190.0(186.0 \text { to } 194.0)^{\star}$ \\
\hline & & $60-69$ & 14536 & $216.0(213.0 \text { to } 220.0)^{\star}$ & $217.0(212.0 \text { to } 222.0)^{\star}$ & $216.0(211.0 \text { to } 221.0)^{\star}$ \\
\hline & & $70-79$ & 9364 & $196.0(192.0 \text { to } 204.0)^{\star}$ & $196.0(191.0 \text { to } 201.0)^{\star}$ & $197.0(191.0 \text { to } 203.0)^{\star}$ \\
\hline & & $80-89$ & 3856 & $149.0(145.0 \text { to } 154.0)^{\star}$ & $145.0(139.0 \text { to } 151.0)^{\star}$ & $157.0(150.0 \text { to } 165.0)^{\star}$ \\
\hline Icen et al (2009), US & & & & & & \\
\hline $1970-2000$ & Dermatologist or & $\geq 18$ & 1633 & $78.9(75.0 \text { to } 82.9)^{\star}+\ddagger$ & $73.2(68.0 \text { to } 78.4)^{\star} † \ddagger$ & $85.5(79.5 \text { to } 91.6)^{\star}+\ddagger$ \\
\hline & physician & $18-29$ & 444 & $77.4^{\star}$ & $75.6^{\star}$ & $79.4^{\star}$ \\
\hline & & $30-39$ & 391 & $81.1^{*}$ & $69.2^{*}$ & $93.3^{*}$ \\
\hline & & $40-49$ & 260 & $71.3^{*}$ & $69.0^{*}$ & $73.6^{\star}$ \\
\hline & & $50-59$ & 230 & $88.0^{\star}$ & $90.7^{\star}$ & $85.2^{\star}$ \\
\hline & & $60-69$ & 174 & $94.2^{\star}$ & $76.2^{\star}$ & $115.3^{*}$ \\
\hline & & $70-79$ & 94 & $73.8^{\star}$ & $71.2^{*}$ & $77.9^{\star}$ \\
\hline & & $\geq 80$ & 40 & $51.4^{\star}$ & $39.8^{\star}$ & $80.0^{\star}$ \\
\hline All ages & & & & & & \\
\hline Schonmann et al (20 & Israel & & & & & \\
\hline 2015 & Dermatologist & $<1$ & - & $23.0^{\star}$ & - & - \\
\hline & & $1-4$ & - & $58.0^{*}$ & - & - \\
\hline & & $5-14$ & - & $117.0^{*}$ & - & - \\
\hline & & $15-24$ & - & $186.0^{\star}$ & - & - \\
\hline & & $25-34$ & - & $315.0^{*}$ & - & - \\
\hline & & $35-44$ & - & $299.0^{*}$ & - & - \\
\hline & & $45-54$ & - & $302.0^{*}$ & - & - \\
\hline & & $55-64$ & - & $347.0^{*}$ & - & - \\
\hline & & $65-74$ & - & $350.0^{*}$ & - & - \\
\hline & & $75-84$ & - & $288.0^{\star}$ & - & - \\
\hline & & $\geq 85$ & - & $173.0^{*}$ & - & - \\
\hline
\end{tabular}

(Continued)

(self-reported, or diagnosis made by a physician or dermatologist) and type of prevalence estimate (period, point, or lifetime prevalence). Specifically, the self-reported lifetime prevalence of psoriasis for the adult population could be almost twice as high (3.77\%, 95\% uncertainty interval $1.32 \%$ to $12.27 \%$ in Australasia, and $3.63 \%, 2.36 \%$ to $5.43 \%$ in western Europe) and up to $1.12 \%$ (0.38\% to $2.84 \%)$ globally, affecting approximately 55.8 million adults worldwide. Supplementary material 2 (eTables 6-11) shows the prevalence estimates for the 21 regions and 189 countries, and according to the type of diagnoses (physician, dermatologist, or self-report) and lifetime prevalence. 


\begin{tabular}{|c|c|c|c|c|c|c|}
\hline \multirow{2}{*}{$\begin{array}{l}\text { Study, country, and } \\
\text { study period }\end{array}$} & \multirow{2}{*}{ Diagnostic method } & \multirow{2}{*}{$\begin{array}{l}\text { Age of } \\
\text { population }\end{array}$} & \multirow{2}{*}{$\begin{array}{l}\text { No with } \\
\text { psoriasis }\end{array}$} & \multicolumn{3}{|c|}{ Incidence rate per 100000 person years $(95 \% \mathrm{Cl})$} \\
\hline & & & & Entire group & Female population & Male population \\
\hline \multicolumn{7}{|c|}{ Znamenskaya et al (2012), Russia } \\
\hline \multirow[t]{4}{*}{2009} & \multirow[t]{12}{*}{ Physician } & $0-18+$ & 99988 & $70.5^{\star}$ & - & - \\
\hline & & $0-14$ & 6069 & $28.8^{\star}$ & - & - \\
\hline & & $15-17$ & 5864 & $118.2^{\star}$ & - & - \\
\hline & & $\geq 18$ & 88055 & $76.0^{\star}$ & - & - \\
\hline \multirow[t]{4}{*}{2010} & & $0-18+$ & 99348 & $69.8^{*}$ & - & - \\
\hline & & $0-14$ & 6045 & $28.2^{\star}$ & - & - \\
\hline & & $15-17$ & 5873 & $128.2^{\star}$ & - & - \\
\hline & & $\geq 18$ & 87430 & $75.4^{*}$ & - & - \\
\hline \multirow[t]{4}{*}{2011} & & $0-18+$ & 99436 & $69.6^{\star}$ & - & - \\
\hline & & $0-14$ & 6104 & $28.0^{\star}$ & - & - \\
\hline & & $15-17$ & 5681 & $126.7^{\star}$ & - & - \\
\hline & & $\geq 18$ & 87651 & $75.2^{\star}$ & - & - \\
\hline \multicolumn{7}{|c|}{ Kubanova et al (2017), Russia } \\
\hline \multirow[t]{4}{*}{2010} & \multirow[t]{28}{*}{ Physician } & $0-18+$ & - & $69.8^{\star}$ & - & - \\
\hline & & $0-14$ & - & $27.9^{\star}$ & - & - \\
\hline & & $15-17$ & - & $127.2^{\star}$ & - & - \\
\hline & & $\geq 18$ & - & $75.4^{\star}$ & - & - \\
\hline \multirow[t]{4}{*}{2011} & & $0-18+$ & - & $69.6^{*}$ & - & - \\
\hline & & $0-14$ & - & $28.0^{\star}$ & - & - \\
\hline & & $15-17$ & - & $126.7^{\star}$ & - & - \\
\hline & & $\geq 18$ & - & $75.2^{\star}$ & - & - \\
\hline 2012 & & $0-18+$ & - & $68.4^{*}$ & - & - \\
\hline & & $0-14$ & - & $27.8^{\star}$ & - & - \\
\hline & & $15-17$ & - & $118.8^{*}$ & - & - \\
\hline & & $\geq 18$ & - & $74.2^{\star}$ & - & - \\
\hline 2013 & & $0-18+$ & - & $65.9^{\star}$ & - & - \\
\hline & & $0-14$ & - & $27.3^{\star}$ & - & - \\
\hline & & $15-17$ & - & $115.6^{*}$ & - & - \\
\hline & & $\geq 18$ & - & $71.7^{\star}$ & - & - \\
\hline 2014 & & $0-18+$ & - & $64.7^{\star}$ & - & - \\
\hline & & $0-14$ & - & $25.1^{\star}$ & - & - \\
\hline & & $15-17$ & - & $108.4^{\star}$ & - & - \\
\hline & & $\geq 18$ & - & $72.4^{\star}$ & - & - \\
\hline 2015 & & $0-18+$ & - & $62.8^{*}$ & - & - \\
\hline & & $0-14$ & - & $24.0^{\star}$ & - & - \\
\hline & & $15-17$ & - & $99.3^{*}$ & - & - \\
\hline & & $\geq 18$ & - & $69.6^{\star}$ & - & - \\
\hline 2016 & & $0-18+$ & - & $65.0^{\star}$ & - & - \\
\hline & & $0-14$ & - & $24.3^{*}$ & - & - \\
\hline & & $15-17$ & - & $95.6^{*}$ & - & - \\
\hline & & $\geq 18$ & - & $72.6^{\star}$ & - & - \\
\hline Huerta et al (2007), U & & & & & & \\
\hline $1996-97$ & Physician & $0-80+$ & 3994 & $140.0^{\star}$ & - & - \\
\hline & & 0-19 & & $116.0^{\star}$ & $121.0^{*}$ & $110.0^{\star}$ \\
\hline & & $20-29$ & & $134.0^{*}$ & $155.0^{*}$ & $111.0^{\star}$ \\
\hline & & 30-39 & & $155.0^{\star}$ & $131.0^{\star}$ & $174.0^{\star}$ \\
\hline & & 40-49 & & $116.0^{\star}$ & $105.0^{\star}$ & $128.0^{\star}$ \\
\hline & & $50-59$ & & $167.0^{\star}$ & $172.0^{*}$ & $161.0^{\star}$ \\
\hline & & 60-69 & & $164.0^{\star}$ & $144.0^{*}$ & $186.0^{\star}$ \\
\hline & & 70-79 & & $163.0^{\star}$ & $118.0^{\star}$ & $224.0^{\star}$ \\
\hline & & $\geq 80$ & & $100.0^{*}$ & $82.0^{*}$ & $173.0^{\star}$ \\
\hline Bell et al (1991), US & & & & & & \\
\hline $1980-83$ & Dermatologist or & $0-70+$ & 132 & $59.9(49.5 \text { to } 70.3)^{*} \dagger$ & $63.6(48.9 \text { to } 78.3)^{\star}+$ & $58.4(42.8 \text { to } 74.1)^{\star} \dagger$ \\
\hline & physician & $<20$ & 21 & $30.9^{\star}$ & $47.1^{\star}$ & $14.8^{\star}$ \\
\hline & & $20-29$ & 25 & $49.1^{\star}$ & $41.3^{*}$ & $59.5^{\star}$ \\
\hline & & 30-39 & 25 & $71.7^{\star}$ & $61.2^{\star}$ & $82.9^{\star}$ \\
\hline & & $40-49$ & 12 & $51.4^{\star}$ & $58.6^{\star}$ & $43.8^{\star}$ \\
\hline & & $50-59$ & 18 & $94.6^{\star}$ & $109.1^{\star}$ & $78.3^{\star}$ \\
\hline & & $60-69$ & 17 & $112.6^{\star}$ & $126.5^{\star}$ & $93.8^{*}$ \\
\hline & & $\geq 70$ & 14 & $77.4^{\star}$ & $54.9^{*}$ & $130.6^{\star}$ \\
\hline
\end{tabular}




\begin{tabular}{|c|c|}
\hline \multicolumn{2}{|c|}{$\begin{array}{l}\text { Table } 4 \text { | Distribution of studies }(n=159) \text { reporting on the } \\
\text { prevalence of psoriasis according to regions }\end{array}$} \\
\hline Region & No of studies* \\
\hline Western Europe & 97 \\
\hline High income North America & 20 \\
\hline East Asia & 16 \\
\hline North Africa and the Middle East & 18 \\
\hline Central Europe & 6 \\
\hline Tropical Latin America & 5 \\
\hline High income Asia Pacific & 4 \\
\hline Australasia & 3 \\
\hline Eastern Europe & 4 \\
\hline South Asia & 3 \\
\hline South East Asia & 1 \\
\hline Eastern sub-Saharan Africa & 1 \\
\hline
\end{tabular}

\section{Discussion}

\section{Main findings}

This systematic review highlighted that epidemiological data on the incidence of psoriasis are limited, with studies conducted mainly in Europe and North America. Findings revealed consistency across studies about the bimodal age pattern of the onset of the disease. No agreement was found on specific sex differences or trends over time.

The prevalence of the disease was highest in high income countries such as Australasia, western Europe, central Europe, and North America. However, the largest adult populations affected by psoriasis lived in the US, India, and China, followed by Germany, Brazil, France, and the UK. Both the incidence and the prevalence of psoriasis had a strong association with age; the disease was less common in children and occurred more frequently in adults.

\section{Strengths and limitation of the study}

The study has several key strengths. Firstly, we undertook an extensive systematic review to search for all available literature since inception by using 11 electronic and regional databases with no restriction on language. Secondly, we used a bayesian framework approach to analyse the data, which is the optimal technique when data are sparse and heterogeneous. ${ }^{14}$ Thirdly, the statistical model was adjusted for potential sources of heterogeneity, such as age strata, type of diagnostic method, and type of prevalence measure used to calculate the prevalence of the disease. Finally, our analysis generated estimates for 21 regions and 189 countries of the world.

Limitations of the study also need to be acknowledged. Firstly, because of limited information on the incidence of psoriasis, it was not possible to include these data in the statistical model. Secondly, in many low income and middle income countries, only small studies with limited information were available, which resulted in greater uncertainty for some estimates. Thirdly, it was not possible to provide estimates by sex and by five year age bands because of the reporting of the data in the included studies; therefore, no age standardised estimates were calculated. However, by using the information on wide age strata we could estimate the prevalence of psoriasis in children, adults, and the overall population. Fourthly, individual studies provided limited information on their sample sizes; therefore, we could not include this information in the statistical model to give more weight to larger studies. Fifthly, the grouping and hierarchy of the countries within region and super region, which aligns with the Global Burden of Disease methods, are mainly based on geography and income. Therefore, these methods might have influenced the income related patterns highlighted in the findings. Finally, because of the complete lack of information for several countries and regions, in some circumstances we could only use estimates of the wider regions or super regions for countries they were nested in. For this reason, estimates for countries with no data might be helpful in guiding policy makers, healthcare practitioners, and patients, but need to be interpreted

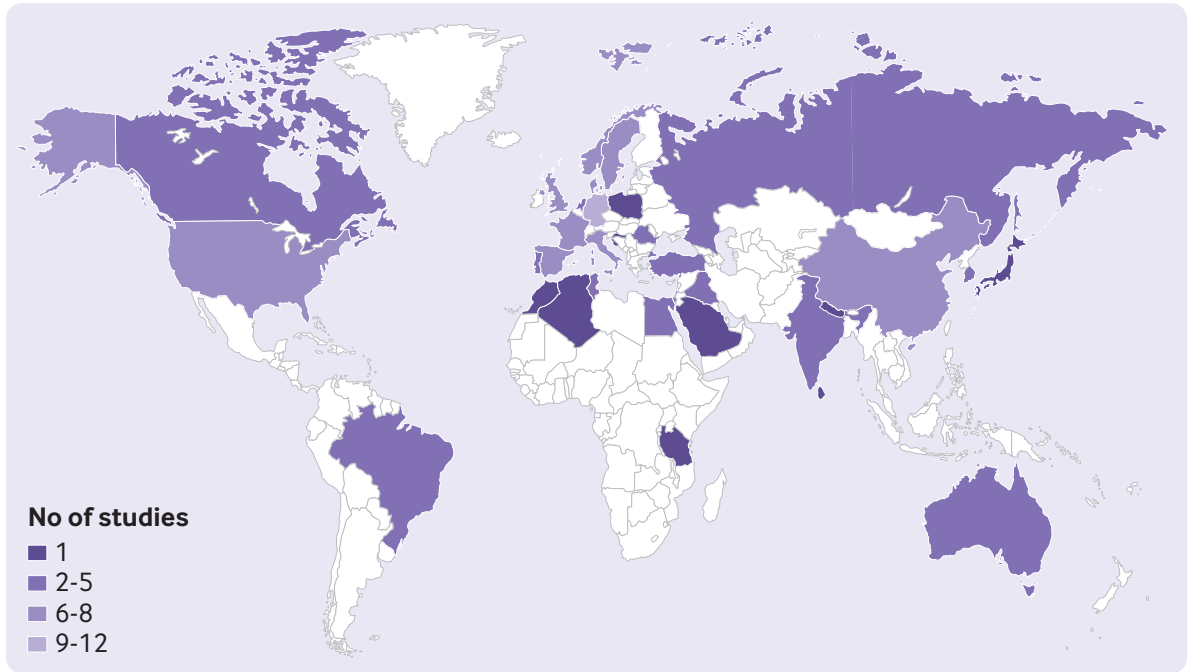

Fig 1 | Distribution of number of studies included in statistical analysis by country. Countries with no observed data are white 


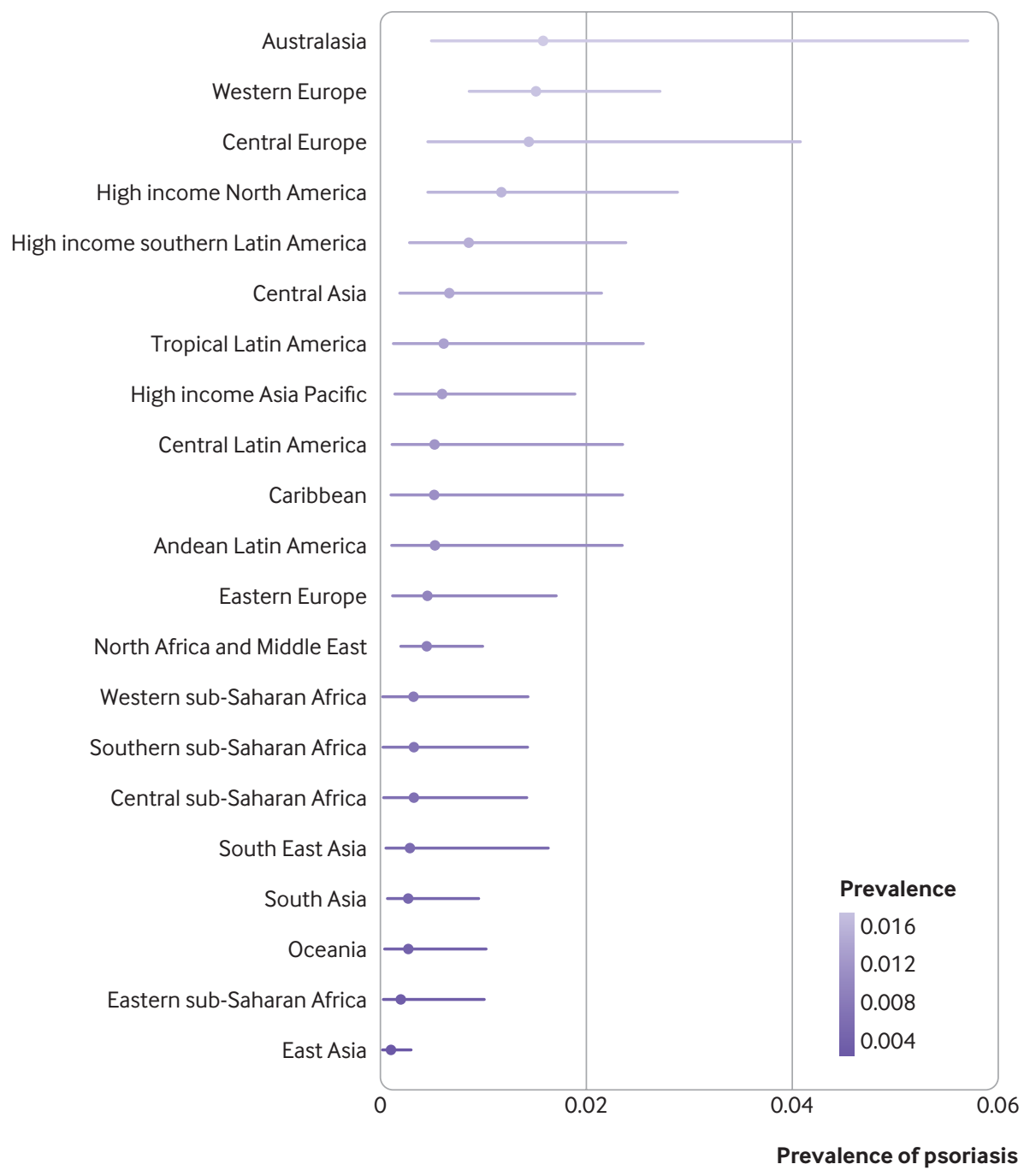

Fig 2 | Crude lifetime (physician or dermatologist diagnosed) prevalence of psoriasis for overall population according to world regions. Regions with observed data: Australasia, central Europe, east Asia, eastern Europe, eastern subSaharan Africa, high income Asia Pacific, high income North America, North Africa and the Middle East, south Asia, South East Asia, tropical Latin America, western Europe. Regions with extrapolated data: central Asia, high income southern Latin America, Caribbean, Andean Latin America, central Latin America, Oceania, central sub-Saharan Africa, southern sub-Saharan Africa, western sub-Saharan Africa

with caution. In particular, these estimates might underestimate or overestimate the true values because they have been extrapolated from the regions or super regions the countries are nested in. Nevertheless, given the long term plan of the Global Psoriasis Atlas, future iterations of the model will include new data that will lead to more accurate estimates of the prevalence of psoriasis, particularly in countries with no observed data currently.

\section{Comparison with other studies}

Research performing similar analyses is limited. ${ }^{11} 12$ Hay and colleagues ${ }^{11}$ provided estimates of the prevalence of psoriasis for 21 regions of the world. Our estimates are lower than their findings, with the main differences in the data sources used. Our systematic review was more extensive and included more recent studies. Furthermore, our statistical model adjusted for important sources of heterogeneity, such as type of diagnostic methods and type of prevalence measure. If we restricted our analysis to the self-reported lifetime prevalence of psoriasis, our estimate of the global prevalence of psoriasis would be similar. ${ }^{12} \mathrm{~A}$ major strength of our research compared with Hay and colleagues ${ }^{11}$ and James and colleagues ${ }^{12}$ is that we were able to provide a measure of the prevalence of psoriasis for 189 countries. Importantly, data included in our study cover the most comprehensive existing scientific literature identified from 11 electronic and regional databases compared with two electronic databases searched in the studies by Hay and colleagues ${ }^{11}$ and James and colleagues. $^{12}$

In contrast to previous suggestions, ${ }^{38}$ we did not find any clear north-south gradient. Conversely, it appeared that the prevalence of psoriasis varied with income, which is similar to the distribution of the disease burden measured as disability adjusted life years (DALYs) by Karimkhani and colleagues. ${ }^{29}$ 


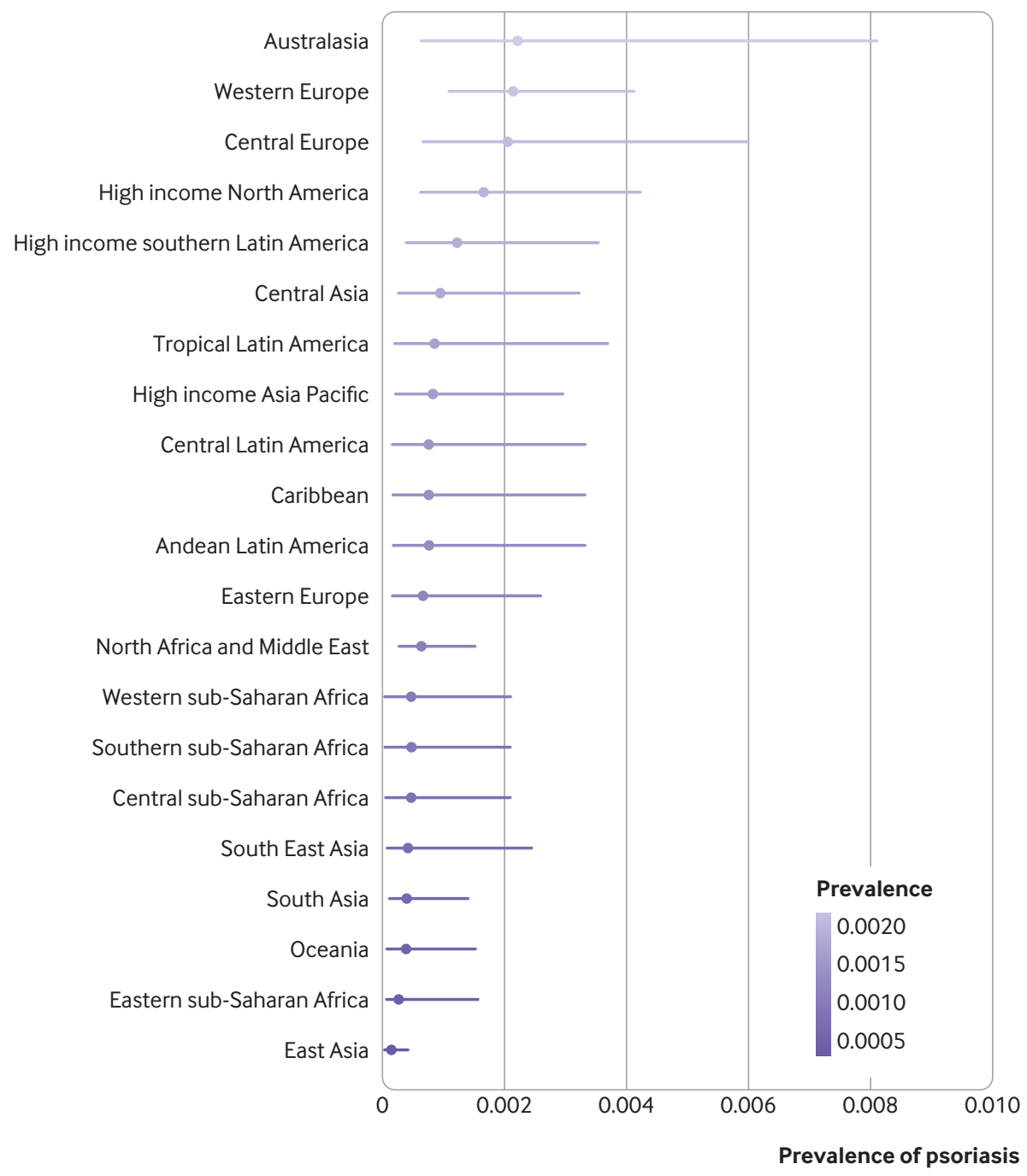

Fig 3 | Crude lifetime (physician or dermatologist diagnosed) prevalence of psoriasis in children according to world regions. Regions with observed data: Australasia, central Europe, east Asia, eastern Europe, eastern sub-Saharan Africa, high income Asia Pacific, high income North America, North Africa and the Middle East, south Asia, South East Asia, tropical Latin America, western Europe. Regions with extrapolated data: central Asia, high income southern Latin America, Caribbean, Andean Latin America, central Latin America, Oceania, central sub-Saharan Africa, southern subSaharan Africa, western sub-Saharan Africa

Countries located in high income regions had a higher prevalence of psoriasis compared with low income countries and regions. There are several possible explanations for this observed pattern. Firstly, the results might be because high income countries have better healthcare systems, increased awareness of the disease, better data quality, and studies from these countries report data from large population based and nationally representative databases. Secondly, the structure of the hierarchical model used was mainly based on geography and income, which might have exacerbated the income related patterns of the findings. Thirdly, high income countries have a larger proportion of older people in the population, which means life expectancy is higher and so there is an increased prevalence of psoriasis. ${ }^{3}$ Finally, the lack of access to healthcare for many people with psoriasis will contribute to an underestimate of its prevalence in many least developed countries.

\section{Interpretation of the findings}

Our study revealed a high country specific variation in the prevalence of psoriasis. Figures were relatively low in regions with young populations, such as south Asia and sub-Saharan Africa, and figures were relatively high in regions with older populations, such as the high income regions. This finding was because of the strong association between the prevalence of psoriasis and age.

Our main analysis reported estimates of the lifetime prevalence of psoriasis according to physician or dermatologist diagnosis. However, these figures might be underestimates of the true prevalence of the disease because most of the data came from studies using databases that only reflect people with psoriasis who sought healthcare; they might not reflect the underdiagnosed population. Conversely, when the analyses reported lifetime prevalence estimates of self-reported psoriasis, the figures were higher in 


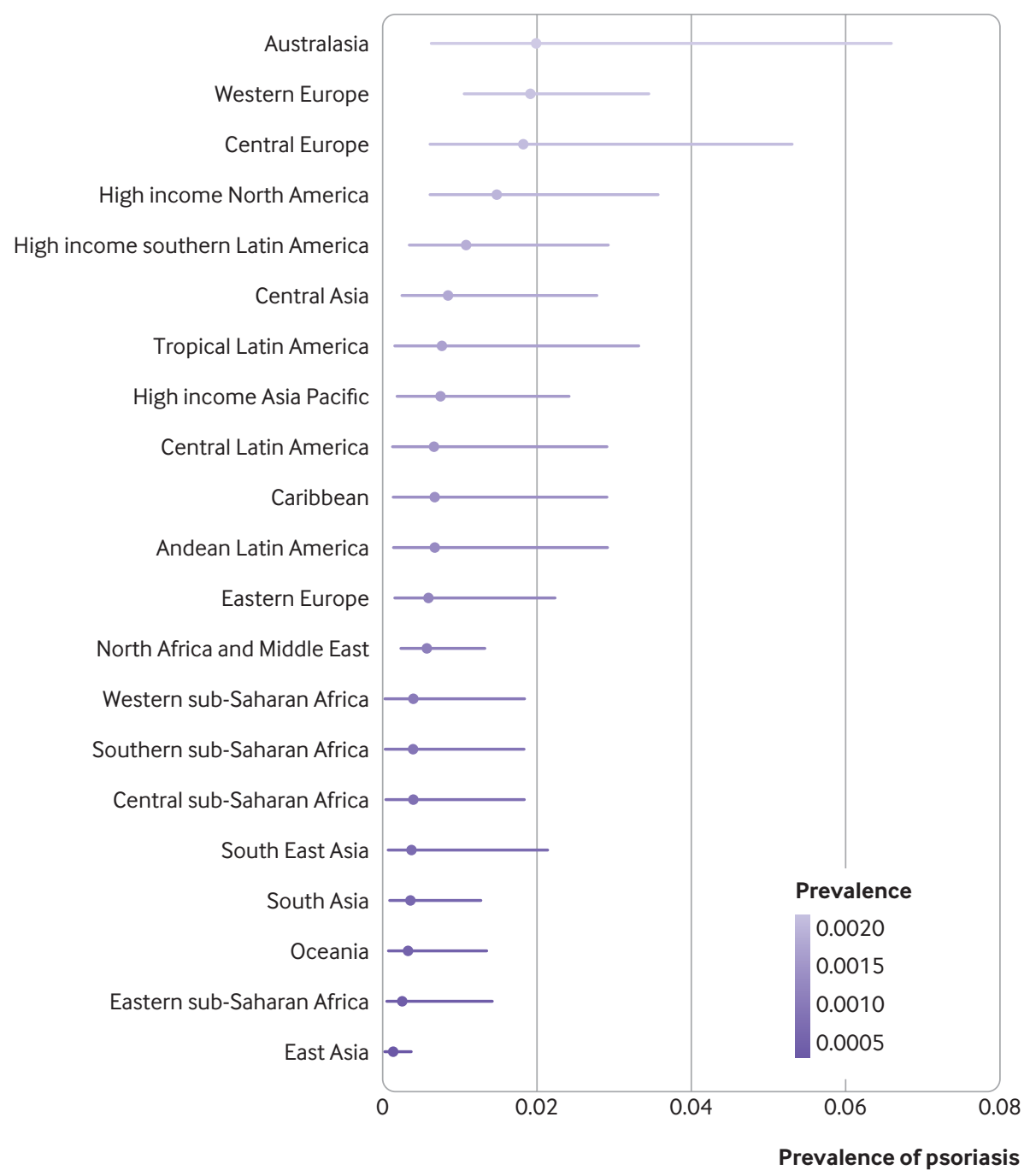

Fig 4 | Crude lifetime (physician or dermatologist diagnosed) prevalence of psoriasis in adults according to world regions. Regions with observed data: Australasia, central Europe, east Asia, eastern Europe, eastern sub-Saharan Africa, high income Asia Pacific, high income North America, North Africa and the Middle East, south Asia, South East Asia, tropical Latin America, western Europe. Regions with extrapolated data: central Asia, high income southern Latin America, Caribbean, Andean Latin America, central Latin America, Oceania, central sub-Saharan Africa, southern subSaharan Africa, western sub-Saharan Africa

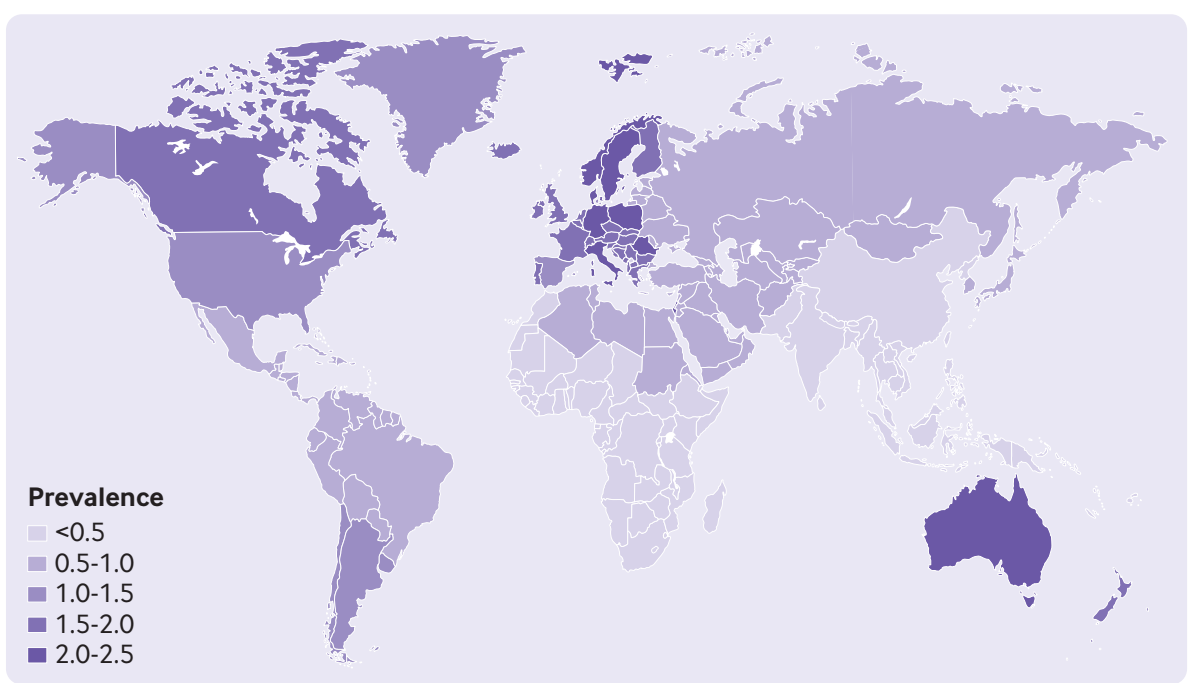

Fig 5 | Lifetime (physician or dermatologist diagnosed) prevalence of psoriasis in adults by country. Details about countries with observed or extrapolated data are given in fig 1 and supplementary material 2 (eTables 6-11) 
comparison, but there was a risk of misdiagnosis of the disease. This finding is consistent with a recent study from Denmark, which found that the prevalence of self-reported psoriasis was higher than the physician reported prevalence. ${ }^{30}$

\section{Conclusions}

Data on the incidence and prevalence of psoriasis have increased in recent years. However, considerable gaps exist in the geographical areas that report this information, particularly from low and middle income countries.

Psoriasis is recognised as a chronic and disabling non-communicable disease, and people affected have a highly visible condition that can be stigmatising. Accounting for population growth and ageing, and the fact that psoriasis mainly affects the adult population, the burden associated with psoriasis could continue to rise.

A clear need exists to improve the quality and increase the amount of data on the epidemiology of psoriasis. Methods, diagnostic criteria, and reporting of the incidence and prevalence of the disease should be standardised. An improved understanding of the epidemiology of psoriasis is important so that resources can be allocated to reduce morbidity, disability, and mortality associated with the disease.

The authors are grateful to Seth Flaxman, Imperial College London, for providing helpful advice on the methods. The authors acknowledge the key role played by the Global Psoriasis Atlas (GPA) Collaborating Organisations in the establishment and organisation of the GPA: the International Psoriasis Council; the International Federation of Psoriasis Associations; and the International League of Dermatological Societies. The views and opinions expressed herein are those of the authors and do not necessarily reflect those of the GPA Collaborating Organisations. The authors are grateful to Rebekah Swan - GPA Programme Manager. Finally, we acknowledge the enthusiastic collaboration of all of the members of the GPA Board of Governors, Steering Committee, regional coordinators and other dermatologists worldwide who provided the data.

Contributors: RP and IYKI are joint first authors. IYKI, RP, DMA, and CEMG led on the conception and design of the study. IYKI and RP searched, extracted, and critically appraised the studies included in the systematic review. RP and EK planned and performed the statistical analyses. IYKI and RP wrote the first draft of the manuscript. $\mathrm{RP}$ and IYKI contributed equally to the study. All authors contributed to the interpretation of the findings and the final draft of the paper. RP is the guarantor. The corresponding author attests that all listed authors meet authorship criteria and that no others meeting the criteria have been omitted.

Funding: The Global Psoriasis Atlas (GPA) project is delivered by the academic project staff based at the University of Manchester and University Medical Center Hamburg-Eppendorf. The GPA is a collaboration between three leading international organisations in the world of dermatology: the International Federation of Psoriasis Associations (IFPA), The International League of Dermatological Societies (ILDS), and the International Psoriasis Council (IPC). The GPA has been supported by grants and sponsorship from the Leo Foundation, Abbvie, Eli Lilly UK, Novartis Pharma AG, UCB, and Almirall. All decisions concerning analysis, interpretation, and publication are made independently of any industrial contribution. DMA and CEMG are funded in part by the National Institute for Health Research (NIHR) Manchester Biomedical Research Centre.

Competing interests: All authors have completed the ICMJE uniform disclosure form at www.icmje.org/coi_disclosure.pdf and declare: support from the Leo Foundation, Abbvie, Eli Lilly UK and Company Ltd, Novartis Pharma AG, UCB, and Almirall for the submitted work; CEMG reports receiving honorariums or research grants from AbbVie, Almirall, Celgene, Eli Lilly, Galderma, Janssen, LEO Pharma, Novartis, Pfizer, Sandoz, Sanofi, and UCB Pharma; DMA reports research grants from AbbVie, Almirall, Celgene, Eli Lilly, Novartis, UCB, and the Leo Foundation; MA reports receiving speakers honorariums or grants from, or participated in clinical trials or health services research projects for Abbott/AbbVie, Almirall, Amgen, Biogen Idec, Boehringer Ingelheim, Celgene, Centocor, Eli Lilly, Forward Pharma, Galderma, GSK, Hexal, Janssen, LEO Pharma, Medac, MSD, Novartis, Pfizer, Sandoz, Teva, TK, Trevi, and Xenoport; no other relationships or activities that could appear to have influenced the submitted work. Ethical approval: Not required.

Data sharing: The raw data are available in the supplementary files.

The lead author affirms that the manuscript is an honest, accurate, and transparent account of the study being reported; and that no important aspects of the study have been omitted; and that any discrepancy from the study as planned have been explained.

Dissemination to participants and related patient and public communities: Findings of the study will be disseminated to patient organisations, specialist and non-specialist audience at international conferences and thorough the Global Psoriasis Atlas website.

Publisher's note: Published maps are provided without any warranty of any kind, either express or implied. BMJ remains neutral with regard to jurisdictional claims in published maps.

This is an Open Access article distributed in accordance with the Creative Commons Attribution Non Commercial (CC BY-NC 4.0) license, which permits others to distribute, remix, adapt, build upon this work non-commercially, and license their derivative works on different terms, provided the original work is properly cited and the use is noncommercial. See: http://creativecommons.org/licenses/by-nc/4.0/.

1 Griffiths CEM, Barker JNWN. Pathogenesis and clinical features of psoriasis. Lancet 2007;370:263-71. doi:10.1016/S01406736(07)61128-3

2 Stern RS, Nijsten T, Feldman SR, Margolis DJ, Rolstad T. Psoriasis is common, carries a substantial burden even when not extensive, and is associated with widespread treatment dissatisfaction. J Investig Dermatol Symp Proc 2004;9:136-9. doi:10.1046/j.1087 0024.2003.09102.x

3 Springate DA, Parisi R, Kontopantelis E, Reeves D, Griffiths CEM, Ashcroft DM. Incidence, prevalence and mortality of patients with psoriasis: a U.K. population-based cohort study. Br J Dermatol 2017;176:650-8. doi:10.1111/bjd.15021

4 Javitz HS, Ward MM, Farber E, Nail L, Vallow SG. The direct cost of care for psoriasis and psoriatic arthritis in the United States. J Am Acad Dermatol 2002;46:850-60. doi:10.1067/mjd.2002.119669

5 Langley RGB, Krueger GG, Griffiths CEM. Psoriasis: epidemiology, clinical features, and quality of life. Ann Rheum Dis 2005;64(Supp 2):ii18-23, discussion ii24-5. doi:10.1136/ard.2004.033217

6 World Health Organization. Global report on psoriasis, 2016. https:// apps.who.int/iris/handle/10665/204417

7 Li R, Sun J, Ren L-M, et al. Epidemiology of eight common rheumatic diseases in China: a large-scale cross-sectional survey in Beijing. Rheumatology (Oxford) 2012:51:721-9. doi:10.1093/ rheumatology/ker370

8 Parisi R, Symmons DPM, Griffiths CEM, Ashcroft DM, Identification and Management of Psoriasis and Associated ComorbidiTy (IMPACT) project team. Global epidemiology of psoriasis: a systematic review of incidence and prevalence. J Invest Dermatol 2013;133:377-85. doi:10.1038/iid.2012.339

9 Enamandram M, Kimball AB. Psoriasis epidemiology: the interplay of genes and the environment. J Invest Dermatol 2013;133:287-9. doi:10.1038/jid.2012.434

10 Farber E, Nall M. Epidemiology: natural history and genetics. Dekker, 1998.

11 Hay RJ, Johns NE, Williams HC, et al. The global burden of skin disease in 2010: an analysis of the prevalence and impact of skin conditions. I Invest Dermatol 2014:134:1527-34. doi:10.1038/jid.2013.446

12 James SL, Abate D, Abate KH, et al, GBD 2017 Disease and Injury Incidence and Prevalence Collaborators. Global, regional, and national incidence, prevalence, and years lived with disability for 354 diseases and injuries for 195 countries and territories, 1990-2017: a systematic analysis for the Global Burden of Disease Study 2017. Lancet 2018;392:1789-858. doi:10.1016/S01406736(18)32279-7

13 Downes MJ, Brennan ML, Williams HC, Dean RS. Development of a critical appraisal tool to assess the quality of cross-sectional studies (AXIS). BMI Open 2016;6:e011458. doi:10.1136/ bmjopen-2016-011458

14 Finucane M, Paciorek C, Danaei G, Ezzati M. Bayesian estimation of population-level trends in measures of health status. Stat Sci 2014;29:18-25. doi:10.1214/13-STS427. 
15 United Nations, Department of Economic and Social Affairs, Population Division. World population prospects: the 2017 revision. Vol II. Demographic Profiles, 2017.

16 Odinets A.The incidence of skin diseases in Stavropol territory in 2010-2016. Klinicheskaya dermatologiya i venerologiya 2017;16. doi:10.17116/klinderma201716632-37.

17 Jacob C, Meier F, Neidhardt K, et al. Epidemiology and costs of psoriasis in Germany - a retrospective claims data analysis. Value Health 2016;19:A566. doi:10.1016/j.jval.2016.09.1269.

18 Kubanova A, Kubanov A, Melekhina L, Bogdanova E. The assessment of the incidence of skin disorders in Russian Federation in 20032016. Vestn Dermatol Venerol 2017:93:22-33.

19 Egeberg A, Skov L, Gislason GH, Thyssen JP, Mallbris L. Incidence and prevalence of psoriasis in Denmark. Acta Derm Venereol 2017;97:808-12. doi:10.2340/00015555-2672

20 Cantarutti A, Donà D, Visentin F, et al, Pedianet. Epidemiology of frequently occurring skin diseases in Italian children from 2006 to 2012: a retrospective, population-based study. Pediatr Dermatol 2015;32:668-78. doi:10.1111/pde.12568

21 Tollefson MM, Crowson CS, McEvoy MT, Maradit Kremers H. Incidence of psoriasis in children: a population-based study. J Am Acad Dermatol 2010;62:979-87. doi:10.1016/j.jaad.2009.07.029

22 Wei JC-C, Shi L-H, Huang J-Y, Wu X-F, Wu R, Chiou J-Y. Epidemiology and medication pattern change of psoriatic diseases in Taiwan from 2000 to 2013: a nationwide, population-based cohort study. I Rheumatol 2018:45:385-92 doi:10.3899/jrheum.170516

23 Vena GA, Altomare G, Ayala F, et al. Incidence of psoriasis and association with comorbidities in Italy: a 5-year observational study from a national primary care database. Eur / Dermatol 2010;20:593-8.

24 Icen M, Crowson CS, McEvoy MT, Dann FJ, Gabriel SE, Maradit Kremers $H$. Trends in incidence of adult-onset psoriasis over three decades: a population-based study. J Am Acad Dermatol 2009;60:394-401 doi:10.1016/j.jaad.2008.10.062

25 Eder L, Widdifield J, Rosen CF, et al. Trends in the prevalence and incidence of psoriasis and psoriatic arthritis in Ontario, Canada: A population-based study. Arthritis Care Res (Hoboken) 2019;71:1084-91. doi:10.1002/acr.23743

26 Khalid JM, Globe G, Fox KM, Chau D, Maguire A, Chiou C-F. Treatment and referral patterns for psoriasis in United Kingdom primary care: a retrospective cohort study. BMC Dermatol 2013;13:9. doi:10.1186/1471-5945-13-9

27 Huerta C, Rivero E, Rodríguez LAG. Incidence and risk factors for psoriasis in the general population. Arch Dermatol 2007;143:155965. doi:10.1001/archderm.143.12.1559

28 Bell LM, Sedlack R, Beard CM, Perry HO, Michet C), Kurland LT. Incidence of psoriasis in Rochester, Minn, 19801983. Arch Dermatol 1991;127:1184-7. doi:10.1001/ archderm.1991.01680070084010

29 Karimkhani C, Dellavalle RP, Coffeng LE, et al. Global skin disease morbidity and mortality: an update from the Global Burden of Disease Study 2013. JAMA Dermatol 2017;153:406-12. doi:10.1001/jamadermatol.2016.5538

30 Egeberg A, Andersen YMF, Thyssen JP. Prevalence and characteristics of psoriasis in Denmark: findings from the Danish skin cohort. BM Open 2019;9:e028116. doi:10.1136/bmjopen-2018-028116

Web appendix: Supplementary material 1 Web appendix: Supplementary material 2

Web appendix: Supplementary material 3 Web appendix: Supplementary material 4 